\title{
Efficacy and Adverse Events During Janus Kinase Inhibitor Treatment of SAVI Syndrome
}

\author{
Stefano Volpi ${ }^{1,2,3,4}$ (1) Antonella Insalaco ${ }^{5} \cdot$ Roberta Caorsi $^{1,2} \cdot$ Elettra Santori $^{6} \cdot$ Virginia Messia $^{5} \cdot$ Oliviero Sacco $^{7}$. \\ Suzanne Terheggen-Lagro ${ }^{8}$. Fabio Cardinale ${ }^{9}$ - Alessia Scarselli ${ }^{10}$. Claudia Pastorino ${ }^{2}$. Gianmarco Moneta ${ }^{5}$. \\ Giuliana Cangemi ${ }^{11}$. Chiara Passarelli ${ }^{12}$ - Margherita Ricci ${ }^{1}$. Donata Girosi ${ }^{7}$ - Maria Derchi ${ }^{13}$ - Paola Bocca ${ }^{2}$. \\ Andrea Diociaiuti ${ }^{14}$. May El Hachem ${ }^{14}$. Caterina Cancrini ${ }^{10}$ • Paolo Tomà ${ }^{15}$. Claudio Granata ${ }^{16}$. Angelo Ravelli ${ }^{1,3}$. \\ Fabio Candotti ${ }^{6}$. Paolo Picco ${ }^{1} \cdot$ Fabrizio DeBenedetti ${ }^{5}$. Marco Gattorno ${ }^{1,2}$
}

Received: 12 November 2018 / Accepted: 10 May 2019 / Published online: 29 May 2019

(C) Springer Science+Business Media, LLC, part of Springer Nature 2019

\begin{abstract}
Objectives Mutations affecting the TMEM173 gene cause STING-associated vasculopathy with onset in infancy (SAVI). No standard immunosuppressive treatment approach is able to control disease progression in patients with SAVI. We studied the efficacy and safety of targeting type I IFN signaling with the Janus kinase inhibitor, ruxolitinib.

Methods We used DNA sequencing to identify mutations in TMEM173 in patients with peripheral blood type I IFN signature. The JAK1/2 inhibitor ruxolitinib was administered on an off-label basis.

Results We identified three patients with SAVI presenting with skin involvement and progressive severe interstitial lung disease. Indirect echocardiographic signs of pulmonary hypertension were present in one case. Following treatment with ruxolitinib, we observed improvements of respiratory function including increased forced vital capacity in two patients, with discontinuation of oxygen therapy and resolution of echocardiographic abnormalities in one case. Efficacy was persistent in one patient and only transitory in the other two patients. Clinical control of skin complications was obtained, and one patient discontinued steroid
\end{abstract}

Stefano Volpi and Antonella Insalaco contributed equally.

Electronic supplementary material The online version of this article (https://doi.org/10.1007/s10875-019-00645-0) contains supplementary material, which is available to authorized users.

Stefano Volpi

stefanovolpi@gaslini.org

1 U.O.C. Clinica Pediatrica e Reumatologia, IRCCS Istituto Giannina Gaslini, Genoa, Italy

2 Centro per le Malattie Autoinfiammatorie e Immunodeficienze, IRCCS Istituto Giannina Gaslini, Genoa, Italy

3 Universita' Degli Studi di Genova, Genoa, Italy

4 UOSD Centro per le Malattie Autoinfiammatorie e Immunodeficienze, IRCCS Istituto Giannina Gaslini, Genoa, Italy

5 U.O. Reumatologia IRCCS Ospedale Pediatrico Bambino Gesù, Rome, Italy

6 Division of Immunology and Allergy, University Hospital of Lausanne, Lausanne, Switzerland

7 Department of Pediatrics, Pediatric Pulmonology and Allergy Unit and Cystic Fibrosis Center, Istituto Giannina Gaslini, Genoa, Italy
8 Emma Children's Hospital, Amsterdam UMC, Amsterdam, Netherlands

9 Pediatric Unit, Giovanni XXIII Hospital, Bari, Italy

10 University Department of Pediatrics, Unit of Immune and Infectious Diseases, Bambino Gesù Children's Hospital IRCCS, University of Rome Tor Vergata, Rome, Italy

11 Central Laboratory of Analyses, IRCCS Istituto Giannina Gaslini, Genoa, Italy

12 UOC Laboratory of Medical Genetics, IRCCS Children Hospital Bambino Gesù, Rome, Italy

13 Pediatric Cardiology Unit, IRCCS Istituto Giannina Gaslini, Genoa, Italy

14 Dermatology Unit, IRCCS Bambino Gesù Children's Hospital, Rome, Italy

15 Department of Imaging, IRCCS Bambino Gesù Children's Hospital, Rome, Italy

16 Department of Pediatric Radiology, IRCCS Istituto Giannina Gaslini, Genoa, Italy 
treatment. One patient, who presented with kidney involvement, showed resolution of hematuria. One patient experienced increased recurrence of severe viral respiratory infections. Monitoring of peripheral blood type I interferon signature during ruxolitinib treatment did not show a stable decrease.

Conclusions We conclude that targeting type I IFN receptor signaling may represent a promising therapeutic option for a subset of patients with SAVI syndrome and severe lung involvement. However, the occurrence of viral respiratory infection might represent an important cautionary note for the application of such form of treatment.

Keywords Recurrent fever $\cdot$ pulmonary fibrosis $\cdot$ JAK inhibitor

\section{Introduction}

Type I interferonopathies are a new class of disorders linked to the upregulation of type I interferon (IFN) [1, 2]. Stimulator of IFN genes (STING)-associated vasculopathy with onset in infancy (SAVI) is caused by gain of function mutations in TMEM173 [3], which lead to a constitutive production of high levels of type I IFNs without infectious triggers [3-5]. SAVI is characterized by systemic inflammation, chronic anemia, growth failure, cutaneous necrotic lesions, and interstitial lung disease $[3,6]$. Low-titer autoantibodies (i.e., anti-nuclear antibody, anti-cardiolipin antibodies) can also be present. Therapeutic management is challenging: steroids are partially effective; patients respond poorly to disease-modifying therapies, such as methotrexate, mycophenolate mofetil, antimalarials, infliximab, and rituximab [3, 6, 7]. Prognosis is poor especially in patients with a severe lung involvement, with a high mortality in the first two decades of life [3].

In light of the encouraging results obtained with the use of JAK inhibitors in other interferonopathies [8], and the ability of blocking type I IFN pathway activation in SAVI PBMC in vitro [3], a few SAVI patients have been treated with JAK1/2 inhibitors with good responses $[9,10]$.

We describe three SAVI patients with severe pulmonary involvement in which treatment with the JAK1/2 inhibitor, ruxolitinib, led to marked amelioration of disease manifestations that was sustained in one patient, but only transient in the other two.

\section{Patients and Methods}

The local ethics committees of Giannina Gaslini Institute and Ospedale Pediatrico Bambin Gesù approved the study.

IFN signature was performed as described [11] with minor modifications. Briefly, RNA was extracted from whole blood from peripheral venipuncture drawn in PAXgene tubes using PAXgene Blood RNA Kit (Qiagen, Hilden, Germany). cDNA was retrotranscribed using SuperScript ${ }^{\circledR}$ VILO $^{\text {TM }}$ cDNA Synthesis Kit (Invitrogen, Carlsbad, California, USA). Selected IFN-stimulated gene (IFI27, IFI44L, IFIT1, ISG15, RSAD2, SIGLEC1) expression was quantified by real-time
PCR using gene-specific primers and probes (Roche) with the ddCt method relatively to a healthy donor calibrator using $H P R T$ and $G 6 P D$ as reference genes. In case samples were run in different assays, the same healthy donor calibrator was used and a positive control was added to assess inter-assay variability.

Mutations in the TMEM173 gene were assessed by standard Sanger sequencing (P1 and P2) or targeted resequencing using a customized panel and analyzed with the NextSeq sequencing platform (Illumina, San Diego, California, USA) (P3). Sequences are analyzed using a bioinformatics pipeline integrated in the BaseSpace Illumina System, and variants are called by VariantStudio Software. Variants identified by nextgeneration sequencing have been validated by Sanger sequencing.

The dyspnea score was defined as follows: 0: absence of dyspnea; 1: dyspnea only during intense physical activity, such as gymnastics at school; 2: dyspnea on moderate physical activity, such as climbing steps; 3 : dyspnea walking; and 4: dyspnea at rest.

\section{Results}

\section{Clinical Presentation}

Patient 1 (current age 13 years old), born from healthy unrelated parents, presented at the age of eight months with isolated erythematosus-infiltrated skin lesions with pustular evolution followed by scarring, chilblains, and severe nail dystrophy by three years of age (Fig. 1a). Skin biopsy of an active nummular violaceous lesion and a scar resolution of a preexisting lesion revealed granulomatous nodular dermatitis, with deep granulomatous folliculitis and secondary fibrosis. The child presented recurrent episodes of bronchospasm, recurrent fever episodes, and worsening of skin lesions. At the age of eight years, focal thickening of the interlobular septa with areas of ground glass opacities with predominant sub-pleural distribution were evident at chest CT (Fig. 1a). A lung biopsy revealed lymphocytic aggregates in the peribronchial interstitial areas with aspects of capillaritis, and contiguous focal sub-atelectasis with macrophages infiltrating the alveoli (Table 1). Immunological 
Fig. 1 Clinical abnormalities in SAVI patients and response to JAK inhibitor therapy. Images of skin lesions in the three patients are shown in $\mathbf{a}, \mathbf{b}$, and $\mathbf{c}$. CT scan showing interstitial lung disease and ground glass appearance in P1 (a) and dense patchy areas of ground glass opacity (probably correlated to previous pulmonary hemorrhages), which appear centrilobular and lobular mainly in the upper lobes in P3 (c). Pulmonary function test of $\mathrm{P} 1$ (a) and P2 (b) showing a mixed restrictive and obstructive alteration. Response to therapy is shown in the lower panels. Curves represent patients' FVC, with $\mathrm{L} / \mathrm{s}$ in the $y$-axis and $\mathrm{L}$ in the $x$-axis. Straight lines represent expected values by age. In P3 (c), diffuse multifocal regions of ground-glass opacity and cystic airspace in the left lower lobe (arrow) were present six months after therapy. PFT were not performed due to age. d H\&E staining of punch skin biopsy from P3 showing parakeratosis and basal vacuolar degeneration of the epithelium and voluminous dermal interstitial and perivasal neutrophil inflammatory infiltrates without vasculitic damage of vessel walls at different magnifications. $L$ liters, $F V C$ forced vital capacity a
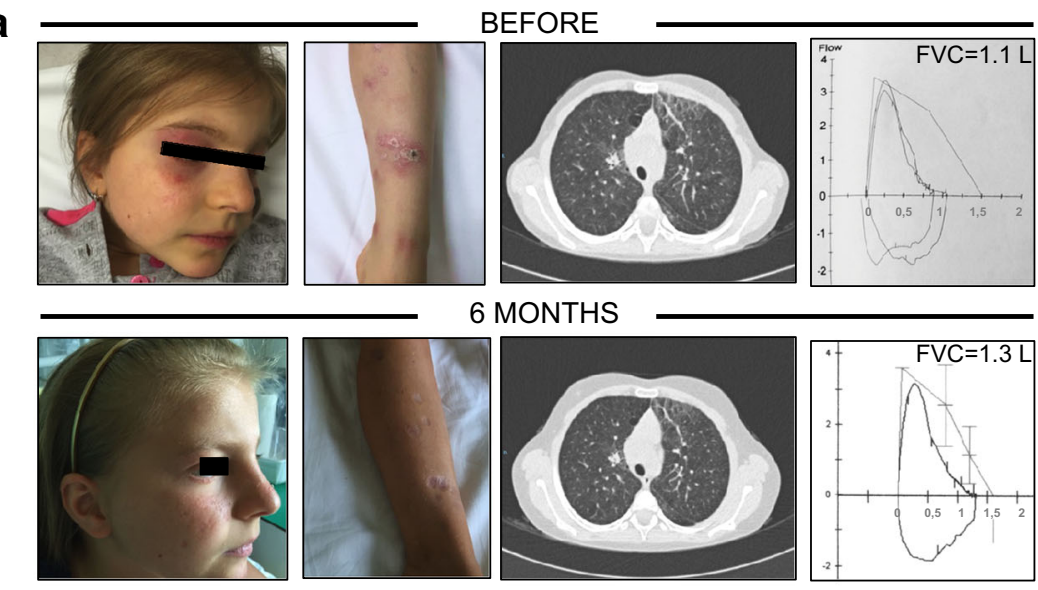

b
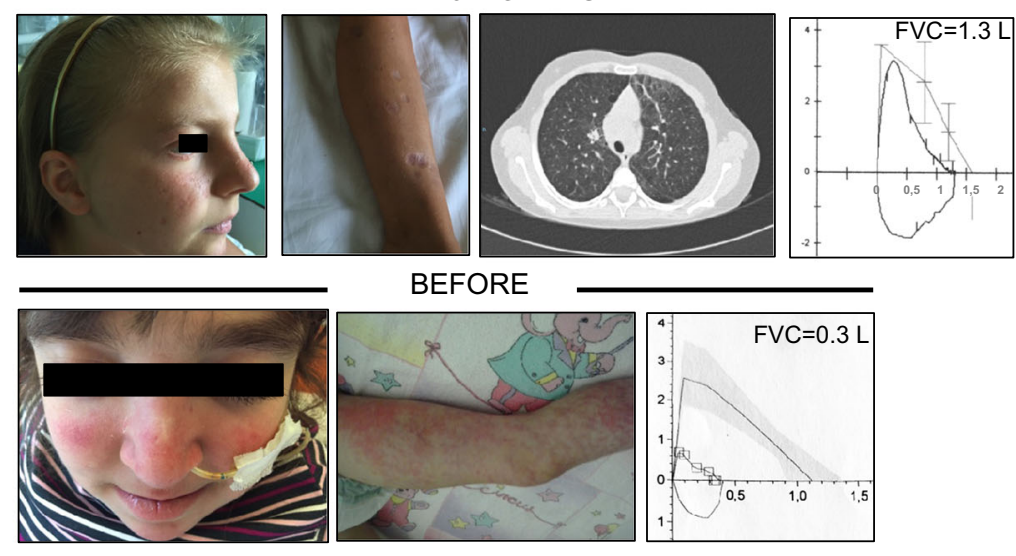

BEFORE
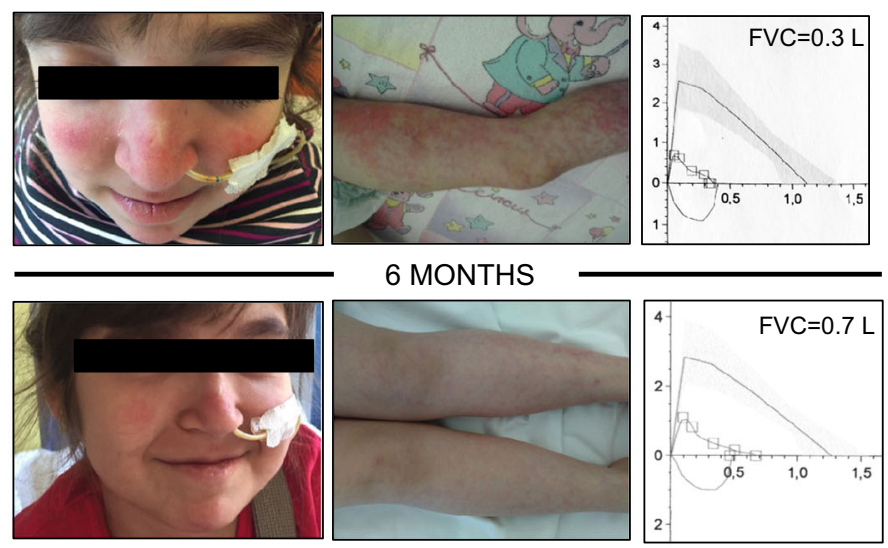

C
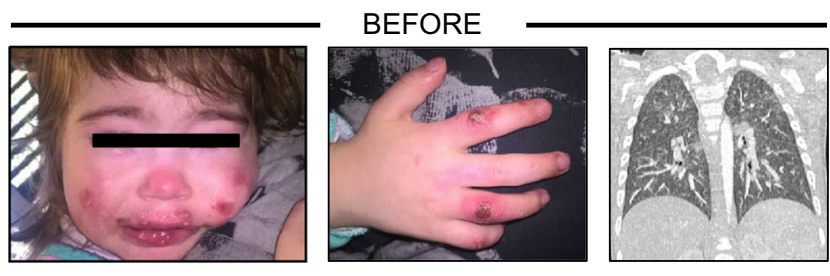

d

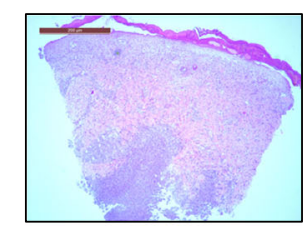

6 MONTHS
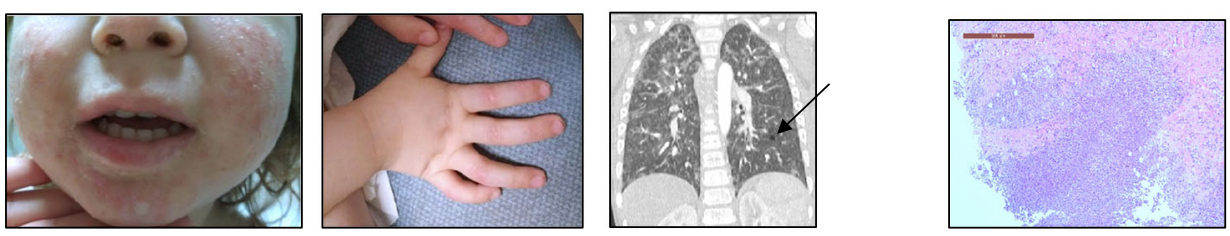

studies revealed increased IgG and IgE, normal lymphocyte subset distribution, and normal lymphocyte proliferation in response to mitogens. Tetanus toxoid-specific IgG were below the protective level when tested at nine years of age (Table 2). Spirometry revealed mixed restrictive and obstructive features (Fig. 1a, Table 3). Differently from the cases described so far, mild renal involvement (microscopic hematuria, mild proteinuria, no biopsy was performed) was also present, associated with hypertension. Steroids (prednisone $1 \mathrm{mg} / \mathrm{kg} /$ day) ameliorated the clinical manifestations with normalization of inflammatory markers. However, disease relapses were observed during steroid tapering. Azathioprine and etanercept were ineffective. Peripheral blood type I IFN signature revealed the activation of type 1 IFN-induced gene expression (Supplementary Fig. 1A). Sanger sequencing of the TMEM173 gene identified a de novo c.463G $>$ A p.Val155Met mutation [14].

Patient 2 (current age ten years old) was recently described [15]. She presented at the age of three months with growth failure and respiratory distress (Table 1). Livedo reticularis was present on the lower limbs (Fig. 1b). The patient required several ICU admissions for ventilator support during lower airway 


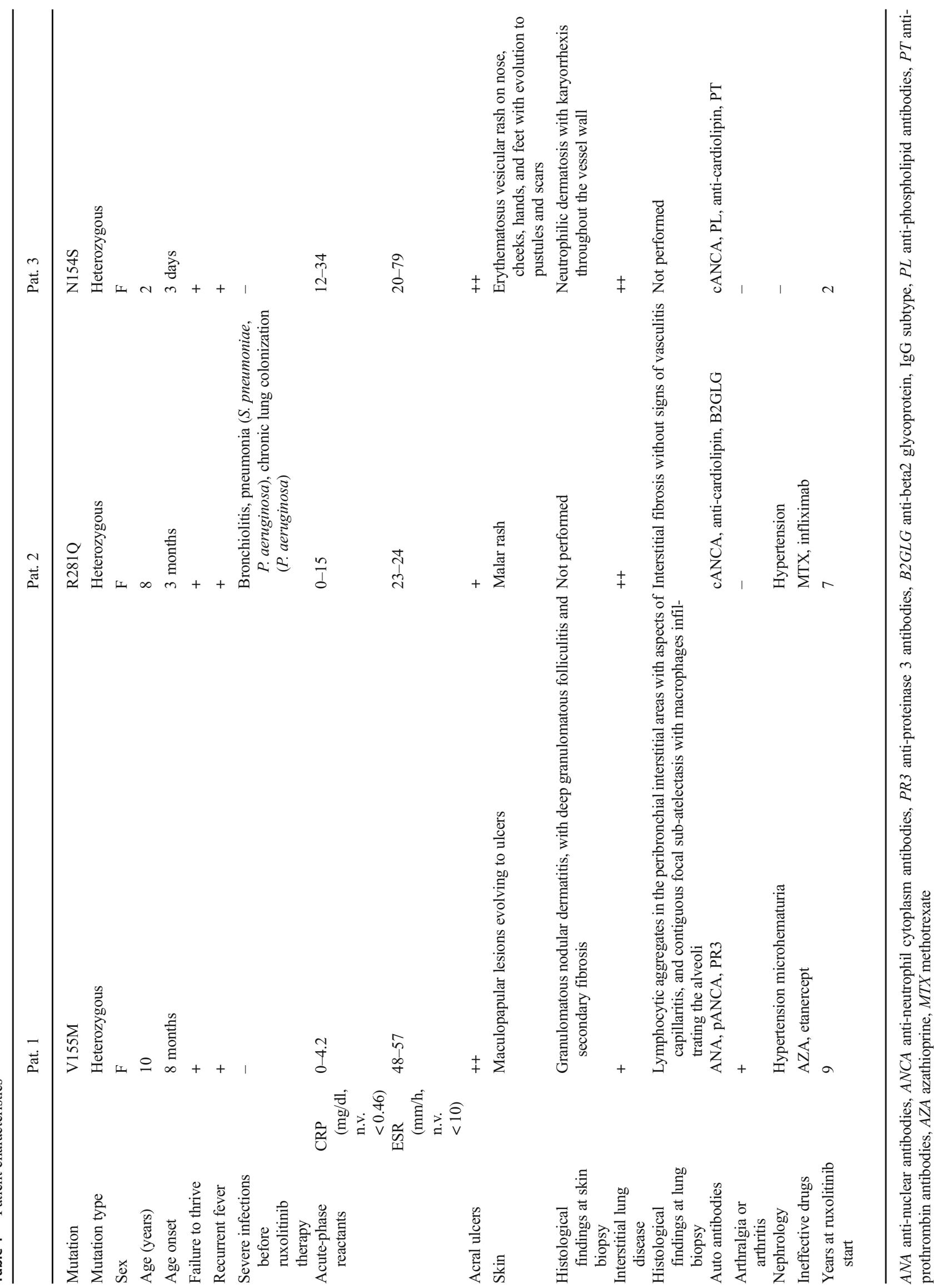




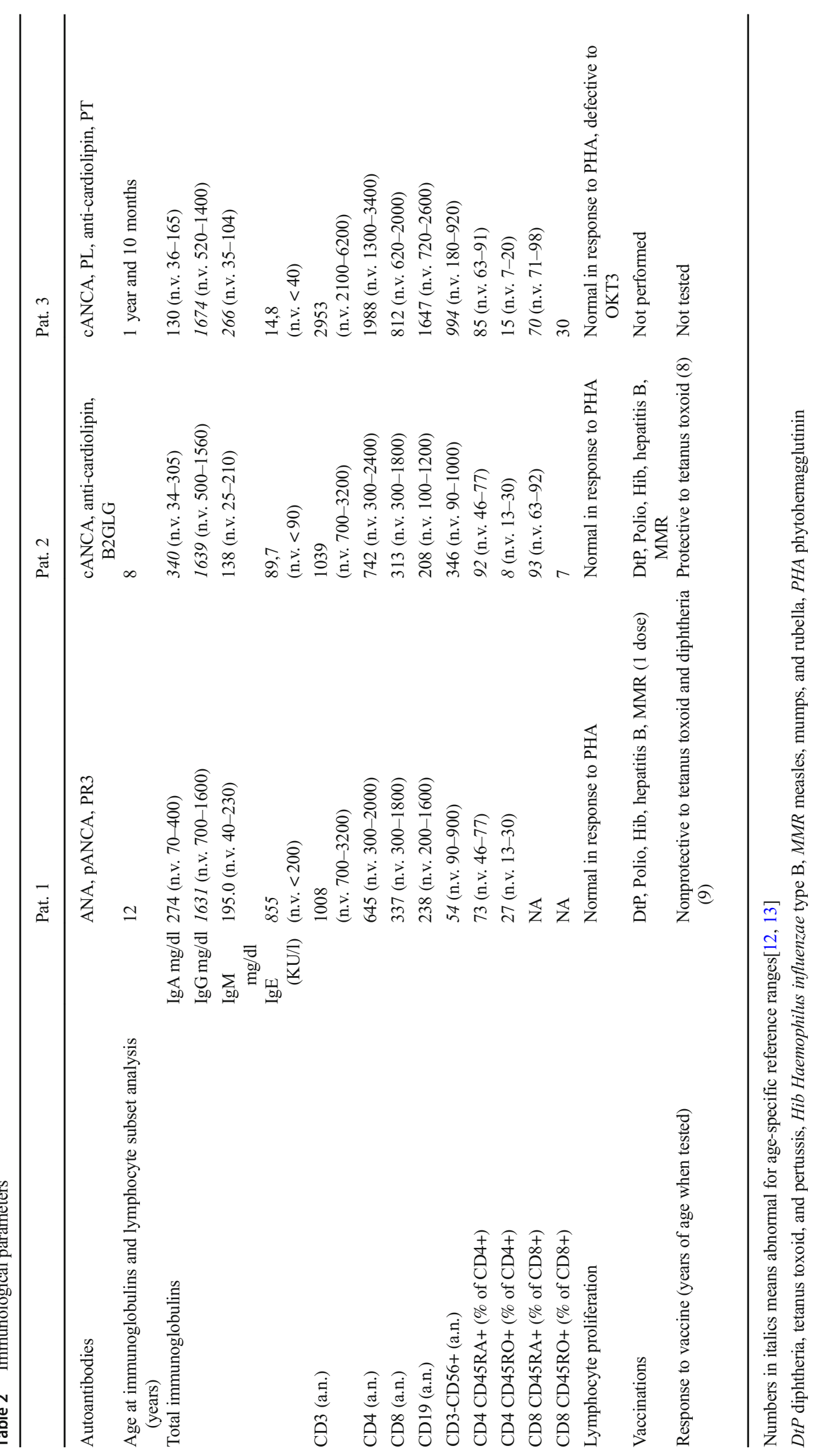


Table 3 Response to therapy

\begin{tabular}{|c|c|c|c|c|c|c|c|c|c|}
\hline Time point (months) & & 0 & 3 & 6 & 10 & 12 & 18 & 23 & 32 \\
\hline \multirow[t]{3}{*}{ Ruxolitinib, mg/day (mg/kg/day) } & $\mathrm{P} 1$ & 0 & $10(0.3)$ & $15(0.5)$ & & $20(0.6)$ & $20(0.6)$ & $20(0.6)$ & $20(0.6)$ \\
\hline & $\mathrm{P} 2$ & 0 & $5(0.25)$ & $10(0.5)$ & & $15(0.7)$ & $5(0.2)$ & & \\
\hline & P3 & 0 & $5(0.45)$ & $15(1.3)$ & & & & & \\
\hline \multirow[t]{3}{*}{ Weight, kg (centile) } & $\mathrm{P} 1$ & $24(5-10)$ & $29(10-25)$ & $29(10-25)$ & & $30(10-25)$ & $32(10-25)$ & $32(5-10)$ & $33(5-10)$ \\
\hline & $\mathrm{P} 2$ & $20(3-5)$ & $21(10-25)$ & $20.4(3-5)$ & & $21.5(10-25)$ & $24.6(10-25)$ & $26(10-25)$ & \\
\hline & P3 & $10(3-10)$ & NA & $11.8(3-25)$ & & & & & \\
\hline \multirow[t]{3}{*}{ Height (centile) } & $\mathrm{P} 1$ & $<3$ & $<3$ & $<3$ & & $<3$ & $<3$ & $<3$ & $<3$ \\
\hline & $\mathrm{P} 2$ & $<3$ & $<3$ & $<3$ & & $<3$ & $<3$ & $<3$ & \\
\hline & $\mathrm{P} 3$ & $3-10$ & NA & $25-50$ & & & & & \\
\hline \multirow[t]{2}{*}{ FVC } & $\mathrm{P} 1$ & 1.1 & 1.2 & 1.3 & & 1.4 & 1.3 & 1.6 & 1.5 \\
\hline & $\mathrm{P} 2$ & 0.3 & 0.6 & 0.7 & & NP & NP & & \\
\hline \multirow[t]{2}{*}{$\mathrm{FVC} \%$} & $\mathrm{P} 1$ & 70 & 84 & 82 & & 87 & 90 & 96 & \\
\hline & P2 & 40 & 54 & 53 & & $\mathrm{NP}$ & $\mathrm{NP}$ & & \\
\hline \multirow[t]{2}{*}{ Sat. $\mathrm{O}_{2} \% 6 \mathrm{MWT}$} & $\mathrm{P} 1$ & ---- & -----. & -------------- & --NP-----. & ----------------. & 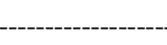 & ------ & \\
\hline & $\mathrm{P} 2$ & $84^{*}$ & 90 & 98 & & NP & NP & & \\
\hline \multirow[t]{2}{*}{ Dyspnea } & $\mathrm{P} 1$ & 3.0 & 0.5 & 0.5 & & 0.5 & 0.5 & 0.5 & 0 \\
\hline & $\mathrm{P} 2$ & 4 & 1 & 1 & & 2 & 3 & & \\
\hline \multirow[t]{3}{*}{ Prednisone (mg/kg/day) } & $\mathrm{P} 1$ & 0.4 & 0.2 & 0.2 & & 0.1 & 0.1 & 0.1 & 0 \\
\hline & $\mathrm{P} 2$ & 0.3 & 0.3 & 0.2 & & & & & \\
\hline & P3 & 0 & 0 & 0 & $2^{* *}$ & & & & \\
\hline \multirow[t]{3}{*}{ CRP (mg/dl) n.v. $<0.4$} & $\mathrm{P} 1$ & 3.6 & 0 & 0.99 & & 0 & 0 & 0 & NP \\
\hline & $\mathrm{P} 2$ & 0.48 & 0.49 & 1.74 & & 0 & 0 & & \\
\hline & P3 & 2.66 & 0.89 & 0.83 & 2.28 & & & & \\
\hline \multirow[t]{3}{*}{$\operatorname{ESR}(\mathrm{mm} / \mathrm{h})$ n.v. $<10$} & $\mathrm{P} 1$ & 55 & 43 & 20 & & 35 & 30 & NP & NP \\
\hline & $\mathrm{P} 2$ & 26 & 32 & 39 & & 24 & 23 & & \\
\hline & P3 & 76 & 36 & 40 & 79 & & & & \\
\hline \multirow[t]{3}{*}{$\mathrm{Hb}(\mathrm{mg} / \mathrm{dl})$} & $\mathrm{P} 1$ & 6.7 & 13.2 & 13.5 & & 12.4 & 12 & 11.7 & 10.6 \\
\hline & $\mathrm{P} 2$ & 12.6 & 13 & 12.9 & & 13.2 & 14.2 & & \\
\hline & P3 & 7 & 8.3 & 9.4 & & & & & \\
\hline \multirow[t]{3}{*}{ Neutrophils (a.n./ $\mu$ l) } & $\mathrm{P} 1$ & 6090 & 3380 & 4250 & & 2160 & 2190 & 3490 & 3100 \\
\hline & $\mathrm{P} 2$ & 7.23 & 7.98 & 7.01 & & 6.98 & 9.220 & 3.87 & \\
\hline & P3 & 6720 & 4520 & 5570 & 5800 & & & & \\
\hline \multirow[t]{3}{*}{ 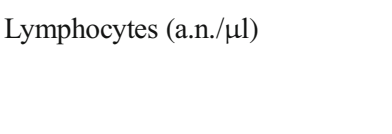 } & $\mathrm{P} 1$ & 2450 & 1780 & 1340 & & 1320 & 1720 & 730 & 960 \\
\hline & $\mathrm{P} 2$ & 900 & 1470 & 1040 & 1670 & 1830 & 820 & 2200 & \\
\hline & P3 & 4530 & 3510 & 1220 & & & & & \\
\hline \multirow[t]{3}{*}{ Platelets $\left(10^{3} / \mu 1\right)$} & $\mathrm{P} 1$ & 473 & 441 & 383 & & 409 & 305 & 355 & 387 \\
\hline & $\mathrm{P} 2$ & 465 & 487 & 478 & 816 & 414 & 440 & 461 & \\
\hline & P3 & 716 & 649 & 766 & & & & & \\
\hline
\end{tabular}

Numbers in italics means abnormal according to age-specific reference ranges

* Interrupted after 4 min for respiratory distress. P3 did not perform respiratory function tests due to his young age

${ }^{* * *}$ Restarted oral prednisone at $2 \mathrm{mg} / \mathrm{kg} /$ day at 10 months because of worsening of the radiological signs of disease at chest CT

$N P$ did not perform, $6 M W T 6$ min walking test, $F V C$ forced vital capacity, $C R P$ C-reactive protein, $E S R$ erythrocyte sedimentation rate, $H b$ hemoglobin

infections (bronchiolitis and pneumonia). CT scan showed extensive ground glass abnormalities (Fig. 1b). Chronic Pseudomonas aeruginosa infection was documented. Lung biopsy showed interstitial fibrosis without signs of vasculitis. Echocardiographic imaging showed indirect signs of pulmonary hypertension and mild dilation of the pulmonary artery. Steroids were only partially effective, and association of methotrexate and infliximab were ineffective, resulting in steroid dependency. Daily oxygen support and non-invasive ventilation at night were required. Cheeks and nose 
telangiectatic skin lesions and unilateral vocal cord paresis were also observed (Fig. 1b, Table 1 and Table 3). Except for the elevation of total IgA and IgG and a mild lymphopenia, immunological studies did not reveal major alterations (Tables 2 and 3). Peripheral blood type I IFN signature was positive (Supplementary Fig. 1A). Targeted sequencing revealed a c.842G $>$ A p.Arg281Gln mutation in TMEM173 [15].

Patient 3 (current age three years old), the only child of healthy, unrelated parents, presented at three days of life, with an erythematosus vesicular rash on the nose and cheeks, later spreading to her hands and feet with evolution to pustules and scars (Fig. 1c and Table 1). She was admitted at the age of three months with recurrent low-grade fever, cough, diarrhea, and dermatitis variably responsive to glucocorticoids and antibiotics in the suspect of a combined immunodeficiency. Skin biopsy was consistent with neutrophilic dermatosis with karyorrhexis throughout the vessel wall (Fig. 1d). Chest CT revealed focal thickening of the interlobular septa with areas of ground glass opacities. Blood investigations revealed microcytic anemia, increased acute-phase reactants, hypergammaglobulinemia, mild reduction of $\mathrm{C} 3$ levels, and low-titer autoantibodies (anti-cardiolipin), with a normal distribution of lymphocyte subsets. Lymphocyte proliferation was normal in response to PHA and low but present in response to OKT3 (Tables 1 and 2). Peripheral blood type I IFN signature was positive (Supplementary Fig. 1A). The patient was started on glucocorticoids ( $1 \mathrm{mg} / \mathrm{kg}$ /day prednisone) with improvement of clinical and laboratory findings, but recurrence of symptoms at tapering. Targeted genome sequencing revealed a de novo heterozygous mutation in TMEM173 c. $461 \mathrm{~A}>\mathrm{G}$ (p.Asn154Ser).

\section{Ruxolitinib Therapy Efficacy, Limits, and Side Effects}

Treatment with ruxolitinib was started initially at $0.25 \mathrm{mg} / \mathrm{kg} /$ day in two doses, and subsequently progressively increased until clinical efficacy was obtained, namely, to $0.65 \mathrm{mg} / \mathrm{kg} /$ day in $\mathrm{P} 1,0.7 \mathrm{mg} / \mathrm{kg} /$ day in $\mathrm{P} 2$, and $1.25 \mathrm{mg} / \mathrm{kg} /$ day in $\mathrm{P} 3$.

All patients showed an initial clinical response starting in the first weeks of treatment and evident after three months (Figs. 1 a and 2 a, Table 3), with amelioration of lung disease (assessed by pulmonary functional tests and CT scans) and resolution of cutaneous lesions that was stable in $\mathrm{P} 1$ and only transient in P2 and P3. Furthermore, P1 showed resolution of the microhematuria and was able to progressively taper steroids and stop them after two years.

P2 also showed amelioration of the skin phenotype, reduction of the dyspnea that correlated with an improvement in the 6-min walking test $\left(\mathrm{O}_{2}\right.$ saturation at the end of the test improved from 84 to $98 \%$ ), and increase in total forced vital capacity (Figs. 1 b and 2 b, Table 3). Furthermore, echocardiographic improvement of the indirect signs of pulmonary hypertension was documented, with normalization of pulmonary artery size.

P3 initially showed a good clinical response with amelioration of skin lesions and radiological lung findings (Fig. 1c, Table 3).

Notably, the clinical responses did not correlate with decreased type I IFN signatures, which improved only transiently in P1 during concomitant treatment with high dose steroids and ruxolitinib (Fig. 2b).

During three years of ruxolitinib treatment, P1 presented three flares of the skin lesions requiring brief cycles of steroids. No severe infections occurred. BK virus in the urines was intermittently detected (viral copies from thousands to 2 million $/ \mathrm{ml}$ ), without BK viremia, nor alteration of kidney function.

P2 (follow-up of 18 months) starting from seven months of therapy, experienced an increase in hospitalization because of respiratory infections, caused by Pseudomonas aeruginosa exacerbation, several episodes of rhinovirus infection, influenza A, varicella, and coronavirus (the last requiring mechanical ventilation and extracorporeal membrane oxygenation for 11 days), prompting the decision of reducing ruxolitinib dosage (Fig. 2c). At 18 months of therapy, CT scan revealed a worsening of the interstitial disease with a ground glass appearance and interlobular thickening (Fig. 1b).

P3 (follow-up of 12 months) after ten months on treatment on ruxolitinib presented clinical and radiological relapse of lung disease requiring glucocorticoid therapy $(2 \mathrm{mg} / \mathrm{kg} / \mathrm{day}$ of prednisone) with a prompt response (Fig. 2a). She never presented viral or bacterial infections since the beginning of therapy. Cell blood counts and ruxolitinib serum levels were regularly monitored (Supplementary Fig. 1B, Supplementary Table 1). No cytopenia was observed during the follow-up (Table 3). All patients are continuing ruxolitinib treatment at the time of writing.

\section{Discussion}

Our report confirms that JAK inhibition is a therapeutic resource worth considering for patients with SAVI, including very young children, as in the case of P3, also taking into account that the disease poorly responds to any other available immune-modifying agents. However, contrary to published reports, we observed several episodes of severe viral infections in one patient, suggesting the possibility that JAK inhibitors might significantly increase the risk of infections possibly in cases of more severe lung involvement or in the presence of genetic modifiers, not investigated in our patient, with consequent deterioration of the lung disease. Interestingly, the most frequent virus isolated was rhinovirus, which may be a direct consequence of effective 
a
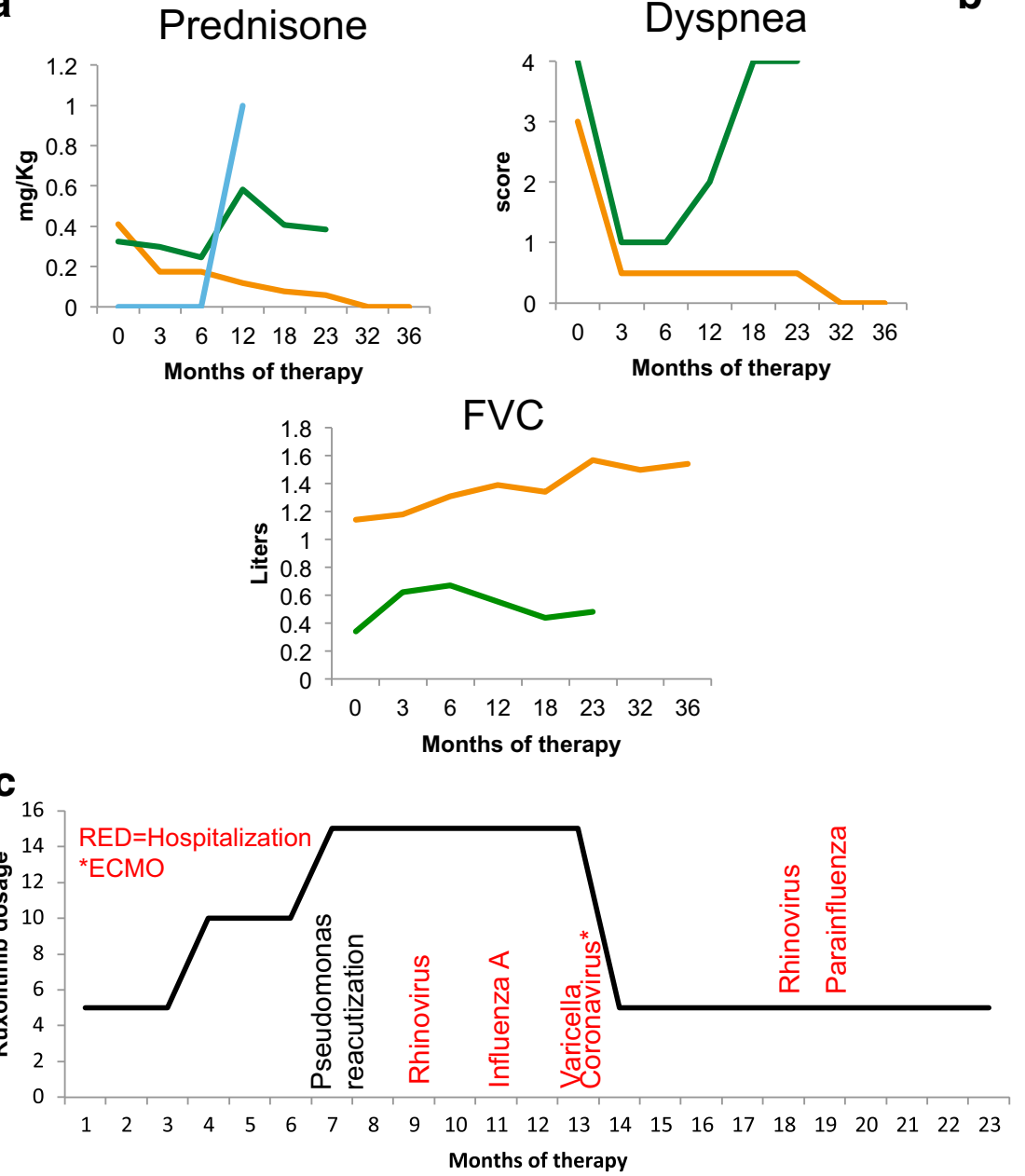

C

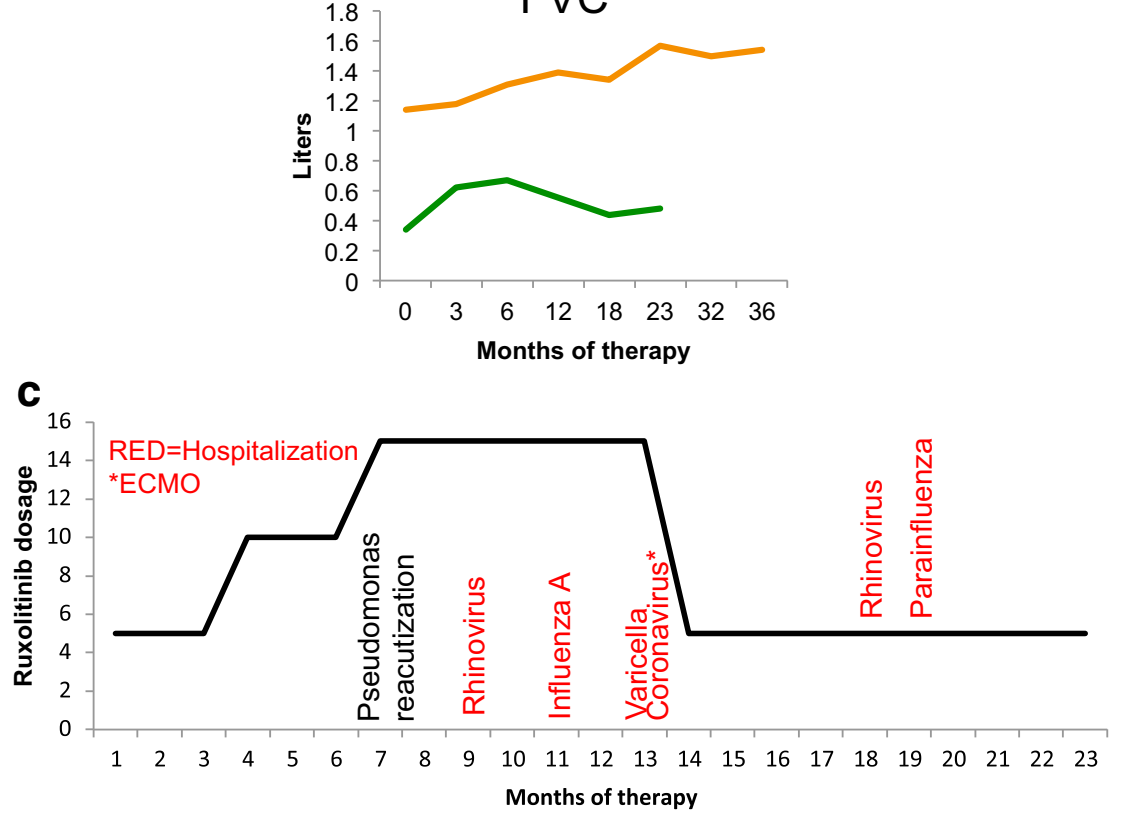

Dyspnea

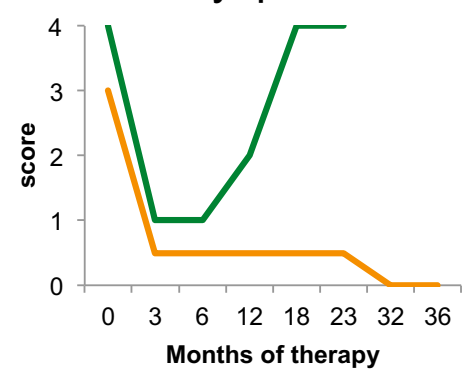

FVC b
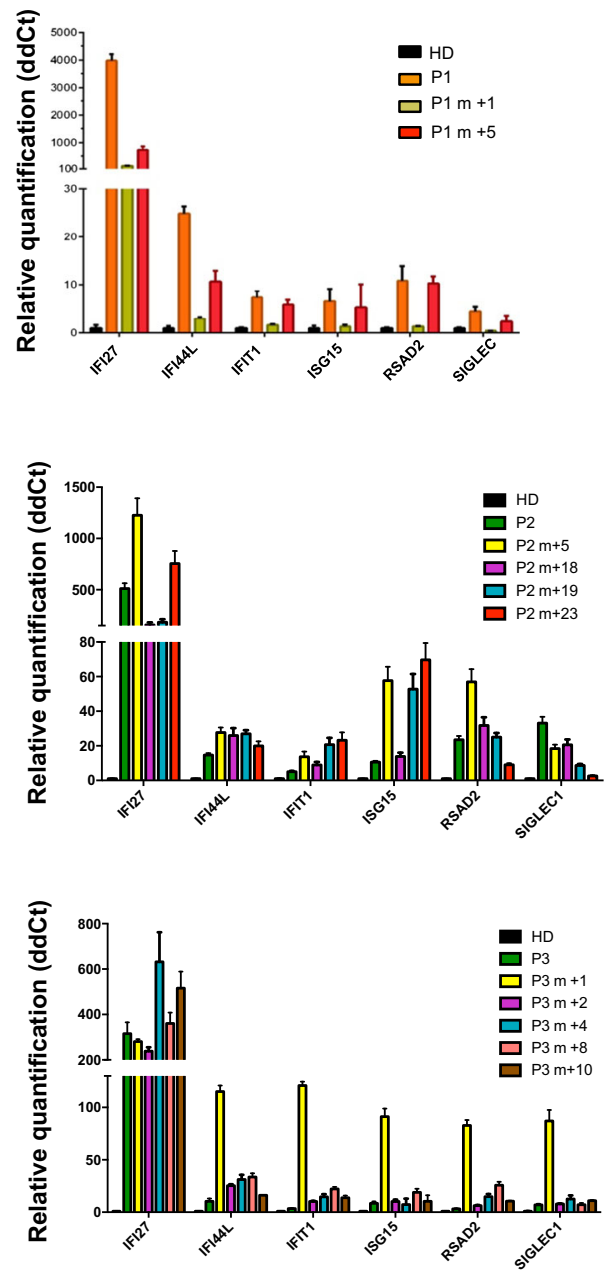

Fig. 2 Response to JAK inhibitor treatment and side effects. Steroid dose and lung disease evolution in response to ruxolitinib therapy (a). Relative quantification of gene expression of six interferon-stimulated genes at baseline and after ruxolitinib treatment; " $\mathrm{m}+$ number" indicates months of therapy (b). Infection occurrence in P2 (c). HD healthy donors, FVC forced vital capacity type I IFN inhibition by ruxolitinib at least in respiratory epithelial cells, where IFN- $\beta$ is required to control rhinovirus $[16,17]$. On the other end, these infections might result from a cumulative effect of the drug with the reported developmental and in vitro proliferative defects of STING mutant $\mathrm{T}$ lymphocytes [18, 19]. Considering the severity of lung disease and the lymphopenia (Table 2) present before ruxolitinib treatment, P2 was started on antibiotic prophylaxis with Bactrim and Azithromycin; that, however, did not seem to prevent the febrile episodes, mostly if not always caused by viral pathogens. Of note, the patient is now on IVIG prophylaxis and no severe infections have been reported so far. Whether this is due to ruxolitinib reduction and/ or IVIG treatment, it is difficult to understand.

We observed a variable medium- and long-term clinical response to the therapy. Only P1 seemed to reach a stable control of the disease, while P2 and P3 required at some point the introduction (P3) or the increase (P2) in steroids to control flares. Several factors might have played a role, including variability of disease severity and inadequate plasma drug levels. Indeed, while all patients were steroid dependent and required several hospital admissions to treat flares, P2 and P3 appear to have a more severe phenotype, with P2 presenting several life-threatening exacerbations requiring admission to ICU and ventilator support during infection episodes and P3 presenting a very early disease onset (three days of life). Additionally, despite dose adjustment and plasma level monitoring, drug dosage appeared to be stably adequate only in P1 (Supplementary Fig. 1 and Supplementary Table 1). Notably, we reduced ruxolitinib dosage in P2 after one year of therapy, in the attempt to prevent infections (Fig. 1c).

Overexpression of a set of interferon-stimulated genes (ISGs) in peripheral blood has consistently been found in all 
reported patients with SAVI syndrome [6, 14, 15]. Modulation of IFN signature following treatment may represent a surrogate biomarker. However, we did not observe a consistent decrease in IFN signature during the treatment. In previous reports of ruxolitinib treatment in patients with SAVI $[9,10$, 20], only a partial or no decrease in IFN signature was observed. The lack of downregulation of IFN signature might be explained by the fast kinetic of JAK inhibition by ruxolitinib as shown by the transient decrease in pSTAT1 following drug intake [9] which seems not to be coupled with a clear impact on ISG expression (Supplementary Fig. 2). Otherwise, it might suggest that the six gene-based test is not appropriate to assess type I interferon activation at least in patients with SAVI treated with a JAK inhibitor.

Finally, it is important to consider that ruxolitinib inhibits not only the signaling of type I interferon receptor but also that of other receptors involved in important inflammatory pathways such as IL6, IL12/23, and interferon $\gamma$. It is possible that the inhibition of some or all of these pathways is responsible for the therapeutic efficacy (and side effects) we see in SAVI.

In conclusion, SAVI disease still appears to be orphan of ideal therapeutic targets. Despite the encouraging results obtained in this and previous reports $[9,10]$, prospective studies with a greater number of patients are warranted to address all the crucial issues linked to JAK inhibitor use in patients with SAVI.

Acknowledgments We thank the patients and their families for their willingness to participate in our study, Drs. Yanick Crow (Imagine Institute, Paris, France) and Benedict Neven (Hôpital Necker-Enfants Malades, Paris, France) for the help in patient diagnosis and advice in the use of ruxolitinib, Dr. Francesca Conti (IRCCS Ospedale Bambin Gesù, Rome, Italy) for patient care, and Dr. Nihel Khoudour (Laboratory of Pharmacology, Université Paris-Est Créteil, France) for serum drug dosage

Authorship Contributions SV designed and performed experiments, acquired and analyzed the data, followed the patients, and wrote the manuscript. AI acquired and analyzed the data, followed a patient, and participated in the writing of the manuscript. RC, AS, FC, VM, OS, STL, FCAR, MR, DG, MD, CC, AR, PP, AD, and MEC acquired the data and followed the patients. CP, ES, GM, PB, GC, and CP conducted the experiments and acquired the data. PT and CG performed and analyzed the radiological exams. FCAN supervised the project and participated in the writing of the manuscript. FDB followed a patient and supervised the project. MG followed the patients, supervised the project, and participated in the writing of the manuscript.

Funding Information SV and M.G. received financial support from Telethon, Italy (grant no. GGP15241A). SV and AR received financial support from the Italian Ministry of Foreign Affairs/Italian Health Ministry (PGR grant IN17GR10). FCAN received combined research support by the University Hospital of Lausanne and the University of Lausanne (CHUV-UNIL).

\section{Compliance with Ethical Standards}

Conflict of Interest The authors declare that they have no conflict of interest.

\section{References}

1. Crow YJ. Type I interferonopathies: a novel set of inborn errors of immunity. Ann N Y Acad Sci. 2011;1238:91-8.

2. Volpi S, Picco P, Caorsi R, Candotti F, Gattorno M. Type I interferonopathies in pediatric rheumatology. Pediatr Rheumatol Online J. 2016;14(1):35.

3. Liu Y, Jesus AA, Marrero B, Yang D, Ramsey SE, Montealegre Sanchez GA, et al. Activated STING in a vascular and pulmonary syndrome. N Engl J Med. 2014;371(6):507-18.

4. Jeremiah N, Neven B, Gentili M, Callebaut I, Maschalidi S, Stolzenberg MC, et al. Inherited STING-activating mutation underlies a familial inflammatory syndrome with lupus-like manifestations. J Clin Invest. 2014;124(12):5516-20.

5. Rodero MP, Decalf J, Bondet V, Hunt D, Rice GI, Werneke $\mathrm{S}$, et al. Detection of interferon alpha protein reveals differential levels and cellular sources in disease. J Exp Med. 2017;214(5):1547-55.

6. Munoz J, Rodiere M, Jeremiah N, Rieux-Laucat F, Oojageer A, Rice GI, et al. Stimulator of interferon genes-associated vasculopathy with onset in infancy: a mimic of childhood granulomatosis with polyangiitis. JAMA Dermatol. 2015;151(8):872-7.

7. Chia J, Eroglu FK, Ozen S, Orhan D, Montealegre-Sanchez G, de Jesus AA, et al. Failure to thrive, interstitial lung disease, and progressive digital necrosis with onset in infancy. J Am Acad Dermatol. 2015

8. Jabbari A, Dai Z, Xing L, Cerise JE, Ramot Y, Berkun Y, et al. Reversal of alopecia areata following treatment with the JAK1/2 inhibitor baricitinib. EBioMedicine. 2015;2(4):351-5.

9. Fremond ML, Rodero MP, Jeremiah N, Belot A, Jeziorski E, Duffy D, et al. Efficacy of the Janus kinase $1 / 2$ inhibitor ruxolitinib in the treatment of vasculopathy associated with TMEM173-activating mutations in 3 children. J Allergy Clin Immunol. 2016;138:1752-5.

10. Sanchez GAM, Reinhardt A, Ramsey S, Wittkowski H, Hashkes PJ, Berkun Y, et al. JAK1/2 inhibition with baricitinib in the treatment of autoinflammatory interferonopathies. J Clin Invest. 2018;128:3041-52.

11. Rice GI, Forte GM, Szynkiewicz M, Chase DS, Aeby A, AbdelHamid MS, et al. Assessment of interferon-related biomarkers in Aicardi-Goutieres syndrome associated with mutations in TREX1, RNASEH2A, RNASEH2B, RNASEH2C, SAMHD1, and ADAR: a case-control study. Lancet Neurol. 2013;12(12):1159-69.

12. Comans-Bitter WM, de Groot R, van den Beemd R, Neijens HJ, Hop WC, Groeneveld K, et al. Immunophenotyping of blood lymphocytes in childhood: reference values for lymphocyte subpopulations. J Pediatr. 1997;130:388-93.

13. Shearer WT, Rosenblatt HM, Gelman RS, Oyomopito R, Plaeger S, Stiehm R, et al. Lymphocyte subsets in healthy children from birth through 18 years of age: the Pediatric AIDS Clinical Trials Group P1009 study. J Allergy Clin Immunol. 2003;112:973-80.

14. Rice GI, Melki I, Fremond ML, Briggs TA, Rodero MP, Kitabayashi N, et al. Assessment of type I interferon signaling in pediatric inflammatory disease. J Clin Immunol. 2017;37(2):12332.

15. Melki I RY, Uggenti C, Van Eyck L,Fremond M, Kitabayashi N, Rice G, Jenkinson E, Boulai A, Jeremiah N, Gattorno M, Volpi S, Sacco O, Terheggen-Lagro S, Tiddens H, Mets I, Morren M, De Haes P, Wouters C, Legius E, Corveleyn A, Rieux-Laucat F, Bodemer C, Callebaut C, Rodero M, and Crow Y. Diseaseassociated mutations identify a novel region in human STING necessary for the control of type I interferon signaling. JACI. 2016.

16. Lamborn IT, Jing H, Zhang Y, Drutman SB, Abbott JK, Munir S, et al. Recurrent rhinovirus infections in a child with inherited MDA5 deficiency. J Exp Med. 2017;214(7):1949-72. 
17. Asgari S, Schlapbach LJ, Anchisi S, Hammer C, Bartha I, Junier T, et al. Severe viral respiratory infections in children with IFIH1 lossof-function mutations. Proc Natl Acad Sci U S A. 2017;114(31): 8342-7.

18. Cerboni S, Jeremiah N, Gentili M, Gehrmann U, Conrad C, Stolzenberg MC, et al. Intrinsic antiproliferative activity of the innate sensor STING in T lymphocytes. J Exp Med. 2017;214(6): 1769-85.

19. Bouis D, Kirstetter P, Arbogast F, Lamon D, Delgado V, Jung S, et al. Severe combined immunodeficiency in stimulator of interferon genes (STING) V154M/wild-type mice. J Allergy Clin Immunol. 2018.

20. Konig N, Fiehn C, Wolf C, Schuster M, Cura Costa E, Tungler V, et al. Familial chilblain lupus due to a gain-of-function mutation in STING. Ann Rheum Dis. 2017;76(2):468-72.

Publisher's Note Springer Nature remains neutral with regard to jurisdictional claims in published maps and institutional affiliations. 